\title{
Upregulated miR-96-5p inhibits cell proliferation by targeting HBEGF in T-cell acute lymphoblastic leukemia cell line
}

\author{
Kaihong Xu' ${ }^{1}$, Xiao Yan ${ }^{1}$, Guifang Ouyang ${ }^{1}$, Jinyi Feng ${ }^{2}$, \\ Lilin $\mathrm{Ye}^{2}$, Xuezhen $\mathrm{Hu}^{3}$, Dingsheng Liu ${ }^{2 *}$
}

${ }^{1}$ Department of Hematology, Ningbo First Hospital, Ningbo City, Zhejiang Province, 315000, China

${ }^{2}$ Department of Oncology and Hematology, Shanghai University of Medicine \& Health Sciences Affiliated Zhoupu Hospital, Shanghai City, 201318, China

${ }^{3}$ Department of Emergency Medicine, The Second Affiliated Hospital and Yuying Children's Hospital of Wenzhou Medical University, Wenzhou City, Zhejiang Province, 325000, China

\begin{abstract}
Introduction. microRNAs (miRNAs) are critical for tumorigenesis and progression of T-cell acute lymphoblastic leukemia (T-ALL). MiR-96-5p has been shown to play important roles in the development of many cancers, but its roles in T-ALL have yet not been studied.

Materials and methods. miR-96-5p expression was detected in T-leukemic cells from peripheral blood of 30 patients with T-ALL using real-time quantitative PCR (RT-qPCR). TargetScan database was utilized to identify the target genes for miR-96-5p, and their target relationship was verified by western blot, dual luciferase reporter assay and RT-qPCR. The effects of miR-96-5p on the viability and proliferation of T-leukemic cells (Jurkat cells) were respectively determined using MTT and BrdU incorporation assays.

Results. miR-96-5p presented low expression levels by qPCR in peripheral blood of T-ALL patients compared to healthy volunteers. Upregulated $\mathrm{miR}-96-5 \mathrm{p}$ by miR-96-5p mimic transfection markedly inhibited the viability and proliferation of Jurkat cells. Furthermore, miR-96-5p negatively regulated the expression of its target gene, $H B E G F$. The decreased viability and proliferation of Jurkat cells caused by miR-96-5p over-expression was suppressed after the introduction of HBEGF plasmid.

Conclusions. The presented study showed that upregulation of miR-96-5p inhibited the viability and proliferation of Jurkat T-leukemic cells through suppressing HBEGF expression. Our study provides a novel sight for understanding the pathological mechanism of T-ALL and suggests that miR-96-5p may be a potential biomarker for the therapy and diagnosis of T-ALL. (Folia Histochemica et Cytobiologica 2020, Vol. 58, No. 3, 219-226)
\end{abstract}

Key words: miR-96-5p; T-cell acute lymphoblastic leukemia; Jurkat T cells; HBEGF

Correspondence address: Dingsheng Liu

Department of Oncology and Hematology,

Shanghai University of Medicine \&

Health Sciences Affiliated Zhoupu Hospital,

No. 1500 Zhouyuan Road, Pudong New District,

Shanghai City, China

phone: +8602168135590

e-mail: QWEok67K@163.com

\section{Introduction}

Acute lymphoblastic leukemia (ALL) is a hematologic malignancy in which the bone marrow produces large numbers of lymphoid progenitor cells. It represents the most common cancer in children, with approximately $60 \%$ of ALL cases in people younger than 20 years [1]. The risk factors for ALL include environmental factors (e.g. X-rays, radiation, alcohol use), genetic factors (e.g. Down syndrome, Bloom syndrome), and infection [2]. ALL cases arise from B-cell or T-cell 
precursors, and are defined as B-cell ALL (B-ALL) and T-cell ALL (T-ALL) [3], respectively. T-ALL accounts for $25 \%$ of ALL cases in adults, and $10-15 \%$ of ALL cases in children [4]. Despite small numbers, T-ALL has achieved lots of attention due to its highly aggressive behavior [5]. Recently, advancements of therapeutic protocols have improved the 5-year overall survival (OS) rate to more than $80 \%$ in children with T-ALL, however, the OS rate in adult T-ALL patients is still low (approximately 50\%) [6]. Furthermore, recurrence is still a huge challenge for all age groups, leading to poor prognosis [6,7]. Hence, further improvements of therapy for T-ALL patients are required.

MicroRNAs (miRNAs) are a class of small (about 22 nucleotides), single-stranded noncoding RNAs $[8,9]$. MiRNAs directly bind to the 3'-untranslated regions (3'-UTRs) of target mRNAs through base-pairing with complementary sequences, thereby regulating gene expression [10]. MiRNAs are relevant to a wide range of cellular functions such as cell differentiation, proliferation, migration, and invasion [11]. Dysfunction of miRNAs has been widely identified in many cancers, including B-ALL and T-ALL $[12,13]$. For instance, miR-101 targets CXCR7 to suppress T-ALL cell invasion and proliferation in vitro and reduce tumor metastasis and growth in vivo [14]. MiR-708 can target CD47 on macrophages to promote phagocytosis, thereby eradicating T-ALL cells [15]. MiR-96-5p can target the caspase-9 gene to inhibit cell apoptosis in hepatocellular carcinoma [16]. Besides, miR-96-5p functions as an oncogenic factor to promote the proliferation of ovarian cancer cells through regulating caveolae1 [17]. MiR-96-5p also serves as a tumor suppressor to repress the breast cancer cell growth and metastasis through modulating the $\mathrm{Wnt} / \beta$-catenin pathway [18]. Nevertheless, the role of miR-96-5p in T-ALL have yet not been described.

The aim of this study was to detect the role and molecular mechanisms of miR-96-5p in T-ALL cells. Here, we firstly determined the expression level of miR-96-5p in T-ALL patients and measured its effects on the proliferation of T-ALL cells. Then, a target gene of miR-96-5p, HBEGF (Heparin-binding epidermal growth factor (EGF)-like growth factor) was identified by the TargetScan database. Next, the interaction of HBEGF gene with miR-96-5p in T-ALL cells was further explored. These experiments may contribute to a better understanding on the pathological mechanism of T-ALL.

\section{Materials and methods}

T-ALL patient blood samples. This experiment was approved by the Ethical Committee of Ningbo First Hospital. Peripheral blood samples from 20 healthy volunteers and
Table 1. The clinicopathologic features of acute lymphoblastic leukemia

\begin{tabular}{|l|c|}
\hline Characteristics & $\begin{array}{c}\text { Number of } \\
\text { patients }\end{array}$ \\
\hline Number & 30 \\
\hline Gender $($ Male/Female) & $9 / 21$ \\
\hline Age (years) & $22.5(16-58)$ \\
\hline $\begin{array}{l}\text { Karyotype }(\text { Normal/Aberrant/No data } \\
\text { available) }\end{array}$ & $12 / 16 / 2$ \\
\hline White blood cell count $\left[\times 10^{9} / \mathrm{L}\right]$ & $12.8(0.4-97)$ \\
\hline Platelet count $\left[\times 10^{9} / \mathrm{L}\right]$ & $215.6(12-587)$ \\
\hline Hemoglobin levels $[\mathrm{g} / \mathrm{L}]$ & $9.25(3.8-18.5)$ \\
\hline
\end{tabular}

Data present median values and ranges.

30 patients with T-ALL were collected at Ningbo First Hospital after obtaining all patients' informed consents.

The diagnosis of T-ALL was based on molecular genetics, cytochemistry, immunology, cytomorphology, and multiparameter flow cytometry. The clinical characteristics of the patients with T-ALL are presented in Table 1.

Isolation of T-leukemic cells. Peripheral blood was collected in tubes with EDTA and separated using the Ficoll density gradient centrifugation method (Ficoll-Histopaque ${ }^{\circledR}$-1077, Sigma-Aldrich, St. Louis, MO, USA). After removing the upper layer, PBMCs (peripheral blood mononuclear cells) were collected and used for the purification of T-leukemic cells. Briefly, PBMCs were resuspended in isolation buffer, heat-inactivated fetal calf serum (FCS, Sigma-Aldrich) was added and incubated with biotinylated anti-human CD235a, CD123, CD56, CD16, CD123, CD19, and CD14 antibodies for 20 min at $4^{\circ} \mathrm{C}$. Besides, Dynabeads (Dynabeads" Untouched" Human T Cells Kit, Thermo Fisher Scientific, Waltham, MA, USA) were washed before use according to the manufacturer's protocol. Next, the cells were centrifuged at $300 \mathrm{~g}$ at $4^{\circ} \mathrm{C}$ for $8 \mathrm{~min}$, resuspended in isolation buffer, pre-washed Depletion Dynabeads (Thermo Fisher Scientific) were added and the cells were incubated at $25^{\circ} \mathrm{C}$ for $15 \mathrm{~min}$, followed by placing in the magnet to obtain the supernatant containing human $\mathrm{T}$ cells.

Cell culture and transfection. The isolated T cells were cultured in RPMI 1640 medium with $15 \%$ FCS, $100 \mu \mathrm{g} / \mathrm{ml}$ streptomycin, 100 Units/ml penicillin, $10 \mathrm{ng} / \mathrm{ml}$ PMA (phorbol 12-myristate 13-acetate), $20 \mathrm{mM}$ HEPES, $2 \mathrm{mM}$ glutamine, 2-ME (mercaptoethanol) (all from Sigma-Aldrich) and $10 \mathrm{ng} / \mathrm{ml}$ interleukin-2 (Peprotech, Rocky Hill, NI, USA) at $37^{\circ} \mathrm{C}$ and in the atmosphere of $5 \% \mathrm{CO}_{2}$.

Jurkat T-leukemia cells were obtained from Cell Bank of the Chinese Academy of Sciences (Shanghai, China). These cells were incubated in RPMI 1640 medium (Gibco) supplemented with $10 \% \mathrm{FCS}, 100 \mu \mathrm{g} / \mathrm{ml}$ streptomycin, 100 Units/ml penicillin, and glutamine $(4 \mathrm{mM})$ at $37^{\circ} \mathrm{C}$, $5 \% \mathrm{CO}_{2}$. 
Table 2. Primer sequences

\begin{tabular}{lr}
\hline Primer & Sequences (5'-3') \\
\hline miR-96-5p forward & ACGATGCACCTGTACGATCA \\
miR-96-5p reverse & TCTTTCAACACGCAGGACAG \\
HBEGF forward & CTGTCTTCGGTCTGCCTCCT \\
HBEGF reverse & AGACCGAAGACAGCACCA \\
U6 forward & GCTTCGGCAGCACATATACTAAAAT \\
U6 reverse & CGCTTCACGAATTTGCGTGTCAT \\
$\beta$-actin forward & TCACCCACACTGTGCCCATCTACGA \\
$\beta$-actin reverse & CAGCGGAACCGCTCATTGCCAATGG \\
\hline
\end{tabular}

MiRNA negative control (miR-NC), miR-96-5p mimic, inhibitor control (NC-inh) and miR-96-5p inhibitor were purchased from Thermo Fisher Scientific. HBEGF plasmid and scramble plasmid were obtained from HANBIO (Shanghai, China). For transfection, Jurkat cells were suspended in Resuspension Buffer R (Neon ${ }^{\circledR}$ Kits, Thermo Fisher Scientific) and mixed with $1 \mu \mathrm{g}$ plasmid, $150 \mathrm{nM}$ miRNA mimics or inhibitor. The mix was taken up into the Neon Pipette tip, plugged into the pipette holder and electroporated by the Neon Transfection System (Thermo Fisher Scientific) based on the protocol from the manufacturer. Then, the cells were added to 24-well plates with medium and cultured for $24 \mathrm{~h}$ in a humidified $5 \% \mathrm{CO}_{2}$ incubator at $37^{\circ} \mathrm{C}$ for further analysis.

Real-time quantitative PCR (RT-qPCR). TRIzol reagent (Invitrogen, Carlsbad, CA, USA) was used to extract total RNA. The isolated RNAs were reverse transcribed into cDNAs as PCR templates with a QuantiTect Reverse Transcription Kit (Qiagen, Hilden, Germany). Each PCR reaction including $\mathrm{PCR}$ templates, $1 \times$ SYBR Master Mix (Applied Biosystems, Foster City, CA, USA) and primers was carried out using an ABI Prism 7500 fast real-time PCR system (Applied Biosystems). The sequence of the primers was listed in Table 2. $\beta$-actin and U6 was used as internal controls. The relative expression of the original target transcript was presented as $2^{-\Delta \Delta \mathrm{Ct}} .{ }^{\Delta \Delta} \mathrm{Ct}=\left(\mathrm{C}_{\mathrm{t}}\right.$ target gene, case group ${ }_{\mathrm{t}}$, reference gene, case group $)-\left(C_{t}\right.$, target gene, control group $-C_{t}$, reference gene, control group $)$

MTT (3-(4,5-Dimethylthiazol-2-yl)-2,5-diphenyltetrazolium bromide) assay. After transfection, the viability of Jurkat cells was evaluated using MTT assay. Jurkat cells $\left(1 \times 10^{6} / \mathrm{ml}\right)$ were inoculated in 96-well plates, followed by incubated for 24 h. Next, MTT (3-(4,5-dimethylthiazol-2-yl)-2,5-diphenyltetrazolium bromide) $(5 \mathrm{mg} / \mathrm{ml}, 10 \mu \mathrm{L})$ was added to the plates followed by incubation with the cells for another $4 \mathrm{~h}$. After centrifugation for $10 \mathrm{~min}$ at $300 \mathrm{~g}$, the supernatant was removed carefully, and DMSO $(100 \mu \mathrm{L})$ was added to each well. The sample's absorbance was assessed at $570 \mathrm{~nm}$ using a microplate spectrophotometer (Thermo Fisher Scientific). $\%$ viable cells $=\left(\mathrm{ABS}_{\text {sample }}-\mathrm{ABS}_{\text {blank }}\right) /\left(\mathrm{ABS}_{\text {control }}-\mathrm{ABS}_{\text {blank }}\right)$ $\times 100 \%$; ABS: Absorbance.
Cell proliferation assay. Jurkat cells after transfection were inoculated in 96-well plates at a density of $1 \times 10^{5}$ cells per ml and cultured in RPMI 1640 medium with $10 \%$ FCS for $72 \mathrm{~h}$. Subsequently, the proliferation of Jurkat cells was evaluated by the colorimetric method using a BrdU Cell Proliferation ELISA kit (Roche, Merck Millipore, Burlington, MA, USA) based on the instruction. The sample's absorbance was examined at $450 \mathrm{~nm}$ in a microplate reader.

Dual luciferase reporter assay. The TargetScan website (http://www.targetscan.org/vert_72/) was utilized to identify the potential binding sits between $H B E G F$ and miR-96-5p. The fragment of HBEGF gene containing wild-type miR96-5p binding sites or the corresponding mutant fragment of HBEGF was cloned into pMIR-REPOR ${ }^{\mathrm{TM}}$ Luciferase vectors (Promega Madison, WI, USA), named as the HBEGFWT reporter vector or the HBEGF-MUT reporter vector. These vectors with miRNA negative control (miR-NC) or miR-96-5p mimic were co-transfected into Jurkat cells by electroporation. In the stage of the pre-experiment, the mRNA expression of HBEGF detected by qPCR was used to present the efficiency of transfection, and the efficiency was high. Next, Jurkat cells were incubated at $37^{\circ} \mathrm{C}$ for $48 \mathrm{~h}$, and subsequently the firefly luciferase activity and Renilla luciferase activity were determined with a Dual-Luciferase ${ }^{\circledR}$ 1000 Assay kit (Promega). The relative luciferase intensity $=$ firefly luciferase activity/Renilla luciferase activity.

Western blot. After transfection, Jurkat cells were lysed with RIPA lysis buffer (Beyotime, Shanghai, China) on ice and centrifuged at $13000 \times g$ to obtain total protein. A BCA Protein Assay Kit (Thermo Fisher Scientific) was applied to assess the protein concentration. Proteins $(20 \mu \mathrm{g})$ were separated on $10 \%$ SDS-PAGE gels through electrophoresis and then electro-transferred onto PVDF membranes. 5\% non-fat milk was utilized to block the membranes at $25^{\circ} \mathrm{C}$ for $1 \mathrm{~h}$. Subsequently, the membranes were incubated with primary antibodies against HBEGF (ab92620, 1/1000 dilution, $\mathrm{ABCAM}$ ) and $\beta$-actin (ab179467, 1/5000 dilution, ABCAM) at $4^{\circ} \mathrm{C}$, washed using TBS buffer with $0.05 \%$ Tween-20, and next cultured with HRP-conjugated secondary antibodies (Goat Anti-Rabbit IgG, \#7074, 1:1000, Cell Signaling Technology Danvers, MA, USA) for $1 \mathrm{~h}$ at room temperature. Protein bands were visualized with a chemiluminescence detection kit (Aidlab Biotechnology, Beijing, China). The semi-quantification of proteins was applied using the ImageJ software, and the relative expression = the protein expression of $\mathrm{HBEGF} /$ the protein expression of $\beta$-actin was determined.

Statistical analysis. Each experiment involving Jurkat cells was repeated three times. Experimental data were expressed as mean $\pm \mathrm{SD}$. The statistics were evaluated by the GraphPad Prism 7 software (San Diego, CA, USA). Student's t test was utilized to identify differences between two groups. 
One-way ANOVA with Bonferroni post hoc comparison test was used for analysis with multiple groups. The significant difference was defined as $p<0.05$.

\section{Results}

\section{Upregulated miR-96-5p inhibits the viability and proliferation of T-leukemic cells}

Firstly, miR-96-5p expression in T-ALL was examined using RT-qPCR. Figure 1 showed that the levels of miR-96-5p in T-leukemic cells from peripheral blood of T-ALL patients were significantly lower compared with that from normal volunteers $(* * p<0.01)$.

Secondly, we detected the effects of miR-96-5p on Jurkat cells. RT-qPCR showed the transfection of miR-96-5p mimic in Jurkat cells considerably up-regulated miR-96-5p expression level as compared to miRNA negative control (miR-NC) (Fig. 2A, $* * * p<0.001)$. MTT assay suggested that miR-96-5p mimic obviously decreased the viability of Jurkat cells in comparison to miR-NC (Fig. 2B, ${ }^{* *}$ p $<0.01$ ).

Furthermore, the proliferation of Jurkat cells was measured by BrdU incorporation assay. The results suggested that transfection of miR-96-5p mimic in Jurkat cells caused an obvious reduction in proliferation as compared to miR-NC transfection (Fig. 2C, $* \mathrm{p}<0.05)$. Thus, miR-96-5p mimics inhibited the viability and proliferation of Jurkat cells.

\section{$H B E G F$ is a target gene of miR-96-5p}

To explore the molecular mechanism of miR-96-5p effects on Jurkat cells, we applied the TargetScan database to predict the potential target genes for miR96-5p. HBEGF (heparin binding EGF like growth factor) was chosen as a candidate gene based on the

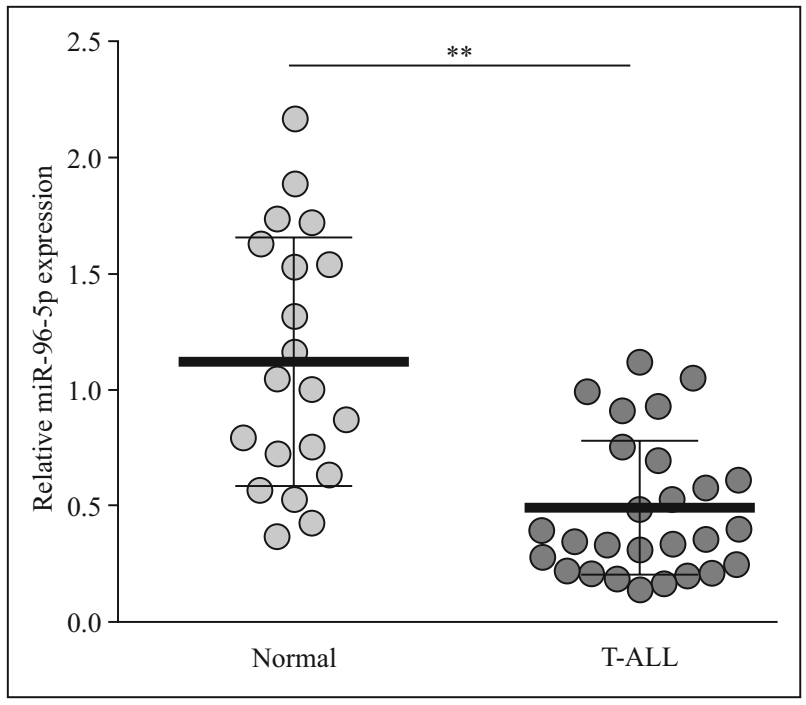

Figure 1. The down-regulated expression of miR-96-5p in T-cell acute lymphoblastic leukemia (T-ALL). RT-qPCR was used to detect the expression of miR-96-5p in T-leukemic cells from peripheral blood of T-ALL patients and healthy volunteers (normal control). ${ }^{* *} p<0.01$.

high ranking in prediction results from the TargetScan database and its critical roles in various cancers [19, 20]. The putative binding sites were listed in Figure $3 \mathrm{~A}$. The interaction of HBEGF with miR-96-5p was validated via dual luciferase reporter assay. The luciferase activity in the HBEGF-WT group was down-regulated by miR-96-5p mimic in comparison to miR-NC $\left({ }^{* *} \mathrm{p}<0.01\right)$, whereas the activity in the HBEGF-MUT group showed no significant difference (Fig. 3B). Thus, miR-96-5p was proved to directly target HBEGF. After Jurkat cells were transfected with in-

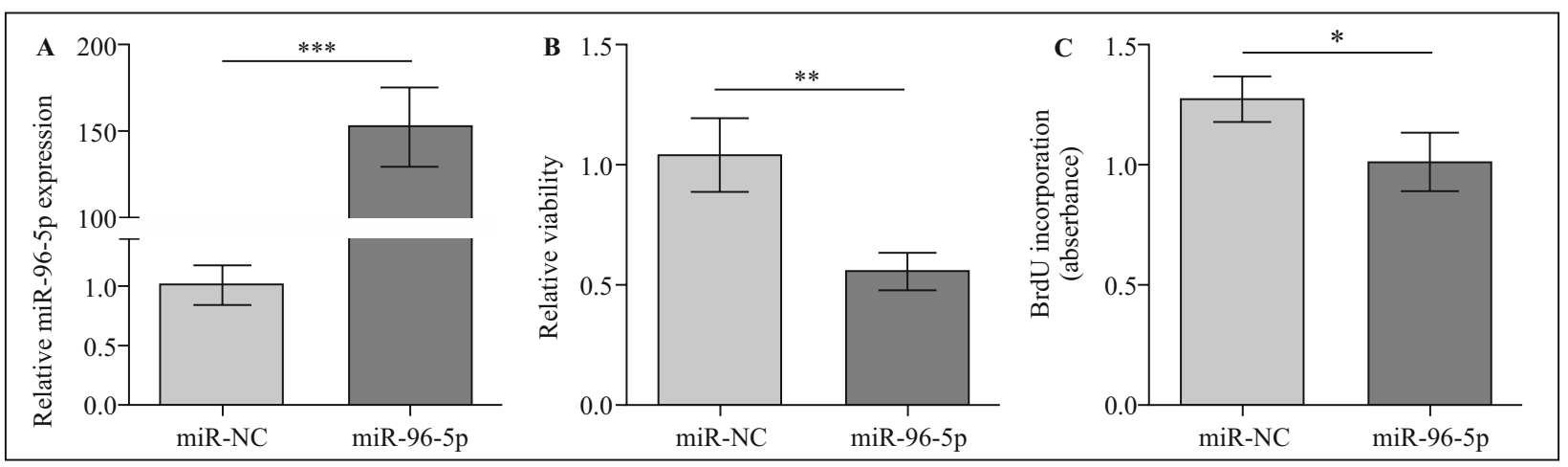

Figure 2. The effect of miR-96-5p on the viability and proliferation of Jurkat cells. A. RT-qPCR showed the expression of miR-96-5p was enhanced by transfection of miR-96-5p mimic as compared to miRNA negative control (miR-NC). B. MTT assay showed that miR-96-5p mimic suppressed the viability of Jurkat cells compared with miR-NC. C. BrdU incorporation assay suggested that the proliferation of Jurkat cells was inhibited by the transfection of miR-96-5p mimic as compared to miR-NC. ${ }^{*} p<0.05,{ }^{* *} p<0.01,{ }^{* * *} p<0.001$. 


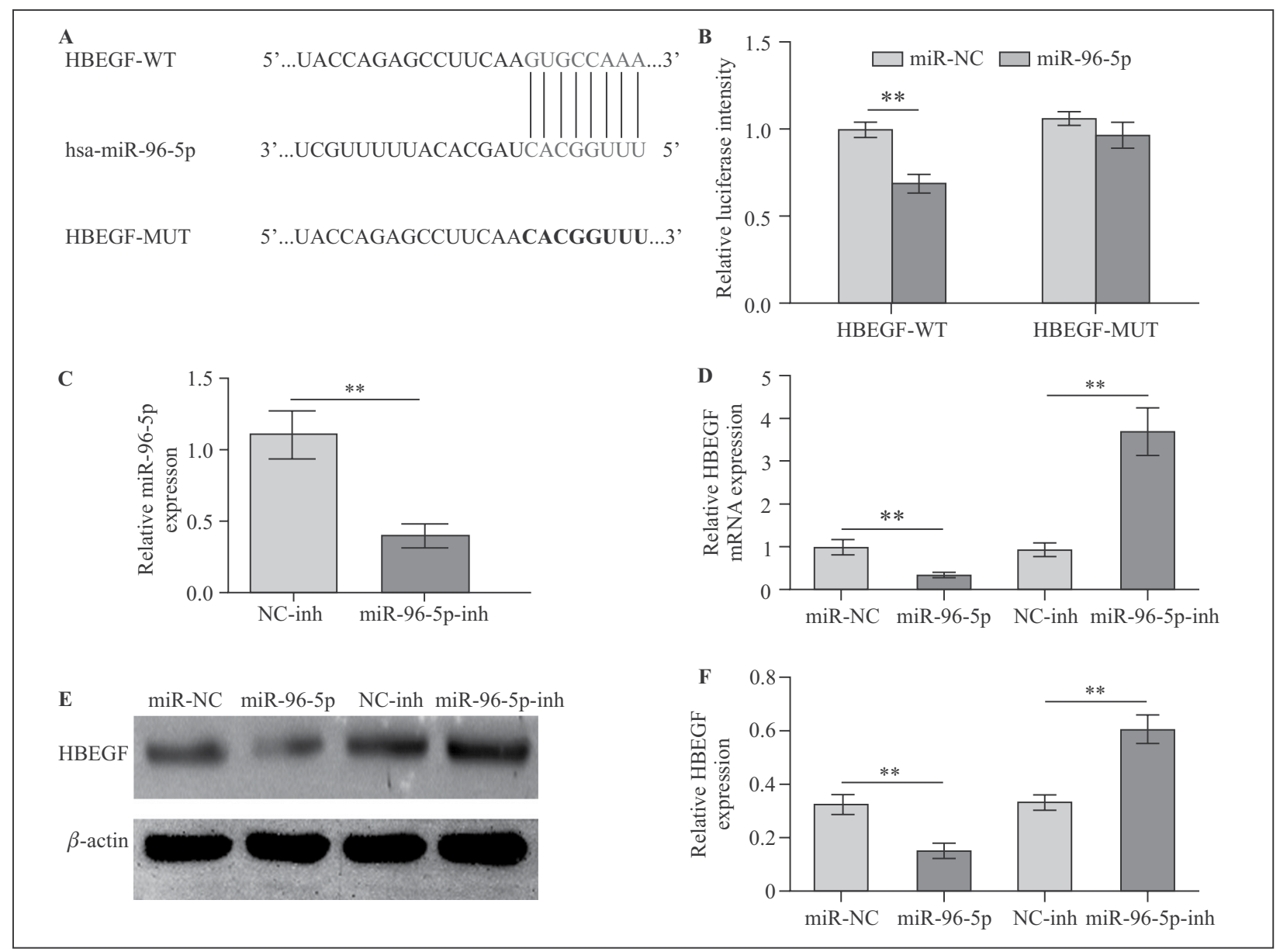

Figure 3. The relationship between miR-96-5p and HBEGF in Jurkat cells. A. The image of the putative binding sites between miR-96-5p and HBEGF. B. Dual luciferase reporter assay was used to verify the target relationship between miR-96-5p and HBEGF. Compared with miR-NC, the interaction between HBEGF-WT vector and miR-96-5p mimic decreased the luciferase activity, whereas the activity had no significant change in the HBEGF-MUT group. C. RT-qPCR suggested the decrease of miR-96-5p expression caused by miR-96-5p inhibitor (miR-96-5p-inh) compared with inhibitor control (NCinh). After Jurkat cells were transfected with miR-96-5p mimic, miRNA control (miR-NC), miR-96-5p-inh or NC-inh. D. RT-qPCR showed HBEGF expression decreased by miR-96-5p mimic compared with miRNA control (miR-NC), but increased by miR-96-5p-inh as compared to NC-inh. E, F. The changing trends of HBEGF expression were further validated by western blot. ${ }^{* *} p<0.01$.

hibitor control (NC-inh) or miR-96-5p inhibitor, miR96-5p inhibitor greatly decreased miR-96-5p expression in comparison to NC-inh (Fig. 3C, ${ }^{* *} \mathrm{p}<0.01$ ). As shown in Figure 3D, compared with miR-NC, miR96-5p mimic markedly down-regulated the mRNA expression levels of HBEGF $(* * \mathrm{p}<0.01)$, and yet miR-96-5p inhibitor up-regulated HBEGF mRNA expression in comparison to $\mathrm{NC}$-inhibitor $\left({ }^{* *} \mathrm{p}<0.01\right)$. Additionally, the protein expression of HBEGF was decreased by miR-96-5 $\mathrm{p}$ mimic as compared to miR$\mathrm{NC}(* * \mathrm{p}<0.01)$, while the protein level of HBEGF was increased by miR-96-5p inhibitor compared with NC-inhibitor $(* * p<0.01$, Figs. 3E and 3F). Collectively, miR-96-5p could directly target HBEGF and negatively regulate HBEGF expression.

\section{Upregulated miR-96-5p inhibits the viability and proliferation of $T$ lymphocytes through regulating $\mathrm{HBEGF}$}

To detect the effects of miR-96-5p/HBEGF on T lymphocytes, Jurkat cells were transfected with miR-96$5 \mathrm{p}$ mimic combined with or without HBEGF plasmid. As shown in Figure 4A, compared with miR-NC+vector, miR-96-5p mimic obviously down-regulated the mRNA expression of HBEGF $(* * p<0.01)$ and HBEGF plasmid up-regulated HBEGF expression $\left({ }^{* *} \mathrm{p}<0.01\right)$. Besides, the co-transfection of miR96-5p mimic with HBEGF plasmid significantly decreased the expression of HBEGF compared with the transfection of HBEGF plasmid alone $(* * p<0.01)$. Furthermore, western blot further confirmed that the 


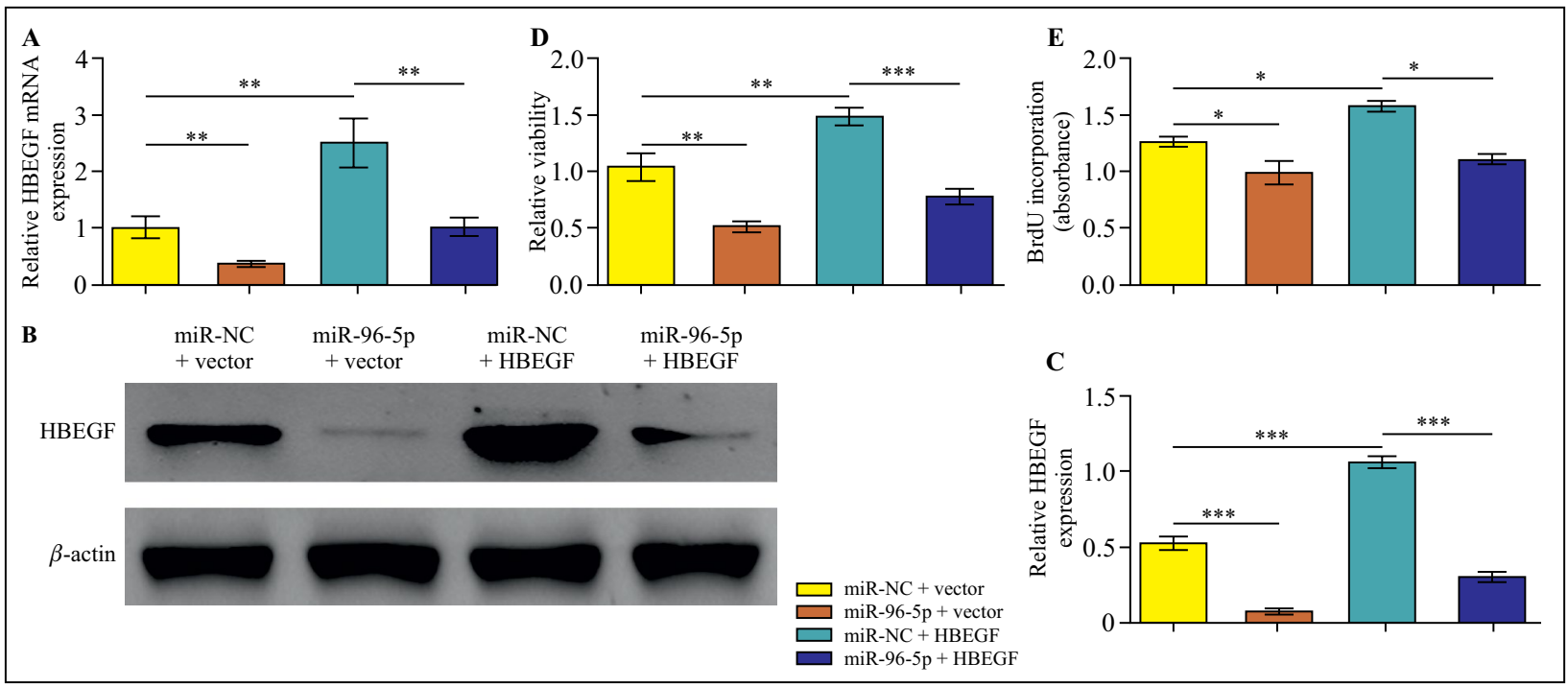

Figure 4. miR-96-5p targets HBEGF to regulate the viability and proliferation of Jurkat cells. Jurkat cells were transfected with miR-96-5p mimic + HBEGF plasmid, miR-96-5p mimic + scramble plasmid (vector), miRNA control (miR-NC) + HBEGF plasmid, or miR-NC + vector. A. RT-qPCR revealed HBEGF expression was increased by HBEGF plasmid and decreased by miR-96-5p mimic compared with miR-NC + vector, and the increase of HBEGF expression induced by HBEGF plasmid was changed by the co-transfection of miR-96-5p mimic with HBEGF plasmid. B, C. The changing trends of HBEGF expression were further validated by western blot. D. MTT assay showed the viability of Jurkat cells was inhibited by miR-96-5p mimic and enhanced by HBEGF plasmid compared with miR-NC + vector, and the enhancement in the cell viability caused by HBEGF plasmid was weakened by the co-transfection of miR-96-5p mimic with HBEGF plasmid. E. BrdU incorporation assay indicated that the cell proliferation had the same change tendency with (D) after Jurkat cells were transfected with those vectors. ${ }^{*} p<0.05$, ** $\mathrm{p}<0.01,{ }^{* * *} p<0.001$.

increase of HBEGF protein expression induced by HBEGF plasmid was weakened by the introduction of miR-96-5p mimic $(* * * p<0.001$, Figs. 4B and $4 \mathrm{C})$. Moreover, the viability of Jurkat cells was markedly enhanced by HBEGF plasmid compared with miR-NC+vector $(* * p<0.01)$, which was weakened by the introduction of miR-96-5p mimic (Fig. 4D). Furthermore, HBEGF plasmid promoted the proliferation of Jurkat cells as compared to miR-NC+vector $\left({ }^{*} \mathrm{p}<0.05\right)$, and yet this promotive effect was suppressed by the co-transfection of miR-96-5p mimic with HBEGF plasmid $\left({ }^{*} \mathrm{p}<0.05\right)$ (Fig. $\left.4 \mathrm{E}\right)$. Therefore, upregulated miR-96-5p inhibited the viability and proliferation of $\mathrm{T}$ lymphocytes through suppressing HBEGF expression.

\section{Discussion}

Despite the remarkable improvements in the treatment of T-ALL, approximately $20 \%$ children with T-ALL and 50\% adult T-ALL patients still fail firstline therapy [6]. Especially, the recurrence result in a very poor prognosis in T-ALL patients [7]. It is reported that T-ALL is relevant to large numbers of genetic abnormalities, which facilitate the development of malignant lymphoid progenitors [21]. Thus, exploring the detailed molecular mechanism of T-ALL is important for improving therapeutic methods. MiRNAs have been applied to many clinical and preclinical studies in various diseases, among which some miRNAs have been proved to be closely associated with the pathogenesis of T-ALL [22]. For instance, miR-96-5p functions as a potential oncogenic factor or tumor suppressor in several cancers [16-18]. Gao et al. have shown that miR-96-5p can target CTNND1 to affect the activation of $\mathrm{Wnt} / \beta$-catenin signaling resulting in the inhibition of the proliferation and invasion of breast cancer cells [18]. Zhang et al. have indicated that miR-96-5p negatively regulates SFRP4 to inhibit cervical cancer cell apoptosis and facilitate the cell viability and metastasis [23]. miR96-5p has important functions in the proliferation and survival of B-cell malignancies [24, 25]. Nevertheless, there is no data regarding the roles of miR-96-5p in T-ALL. Thus, our study determined miR-96-5p expression levels in T-leukemic cells from peripheral blood of T-ALL patients and found the patients with aberrantly low expression of miR-96-5p as compared to that from healthy volunteers. Moreover, we used miR-96-5p mimic to upregulate the expression of 
miR-96-5p, which markedly repressed the viability and proliferation of T-leukemic cells. Thus, miR-96-5p, as a new and potent biomarker, might be used for the therapy and diagnosis of T-ALL.

To further explore the molecular mechanisms of miR-96-5p in T-leukemic cells, TargetScan database was used to identify the potential target genes of miR-96-5p, among which HBEGF was chosen as a candidate. Despite the fact that HBEGF had a high ranking in prediction results of the TargetScan database, HBEGF, as a heparin-binding member of the EGF family [26], has been found to be a potent growth factor associated with tumorigenesis [19]. For instance, HBEGF stimulates EGFR expression and induces angiogenesis of bone marrow endothelial cells in multiple myeloma mouse model [27]. HBEGF over-expression promotes the invasion of gastric cancer cells [28]. Moreover, HBEGF has been observed to be over-expressed in T-ALL cells and associate with the cell survival [20]. In the present study, the direct target relationship between HBEGF and miR-96-5p was confirmed by dual luciferase reporter assay. In addition, miR-96-5p was observed to negatively regulate HBEGF expression.

MiRNAs are proved to mediate posttranscriptional regulation of genes to participate in a wide range of biological processes [29]. MiR-96-5p can target CTNND1 to regulate $\mathrm{Wnt} / \beta$-catenin signaling and thus inhibit the invasion and proliferation of breast cancer [18]. MiR-212 represses the metastasis and growth of epithelial ovarian cancer through controlling HBEGF [26]. Regulation of HBEGF by miR-1192 affects the C2C12 cell osteogenic differentiation [30]. Our study also revealed that the promotive effects on the viability and proliferation of T-leukemic cells induced by HBEGF plasmid transfection were suppressed by the introduction of miR-96-5p mimic. Therefore, miR-96$5 p$ could target HBEGF expression to regulate the viability and proliferation of T-leukemic cells.

In conclusion, our study found the aberrantly low expression of miR-96-5p in peripheral blood mononuclear cells of T-ALL patients. Additionally, upregulated miR-96-5p repressed the viability and proliferation of T-leukemic Jurkat cells through controlling HBEGF expression. Thus, miR-96-5p may be a potential biomarker. These findings contribute to a better understanding of the molecular mechanism of the functional role of miR-96-5p in T-ALL.

\section{Funding}

This work was supported by Natural Science Foundation of Ningbo Municipality (Grant No. 2017A610210 (Grant No.2017A610177) and Discipline Construc- tion Program of Pudong New District Health Bureau of Shanghai (Grant No. PWZzk2017-31).

\section{Competing interests}

The authors state that there are no conflicts of interest.

\section{Contribution of authors}

KX and DL designed the study, supervised the data collection, analyzed the data, XY interpreted the data and prepare the manuscript for publication, and JF, LY and XH supervised the data collection, analyzed the data and reviewed the draft of the manuscript. All authors have read and approved the manuscript.

\section{Availability of data and materials}

All data generated or analyzed during this study are included in this published article.

\section{References}

1. Malard F, Mohty M. Acute lymphoblastic leukaemia. The Lancet. 2020; 395(10230): 1146-1162, doi: 10.1016/s01406736(19)33018-1, indexed in Pubmed: 32247396 .

2. Jacobson S, Tedder M, Eggert J. Adult acute lymphoblastic leukemia: a genetic overview and application to clinical practice. Clin J Oncol Nurs. 2016; 20(6): E147-E154, doi: 10.1188/16.CJON.E147-E154, indexed in Pubmed: 27857258.

3. Follini E, Marchesini M, Roti G. Strategies to overcome resistance mechanisms in T-cell acute lymphoblastic leukemia. Int J Mol Sci. 2019; 20(12), doi: 10.3390/ijms20123021, indexed in Pubmed: 31226848.

4. Bongiovanni D, Saccomani V, Piovan E. Aberrant signaling pathways in T-cell acute lymphoblastic leukemia. Int J Mol Sci. 2017; 18(9), doi: 10.3390/ijms18091904, indexed in Pubmed: 28872614.

5. Drobna M, Szarzyńska-Zawadzka B, Dawidowska M. T-cell acute lymphoblastic leukemia from miRNA perspective: Basic concepts, experimental approaches, and potential biomarkers. Blood Rev. 2018; 32(6): 457-472, doi: 10.1016/j. blre.2018.04.003, indexed in Pubmed: 29703513.

6. Evangelisti C, Chiarini F, McCubrey JA, et al. Therapeutic targeting of mTOR in T-cell acute lymphoblastic leukemia: An Update. Int J Mol Sci. 2018; 19(7), doi: 10.3390/ ijms19071878, indexed in Pubmed: 29949919.

7. Maude SL, Teachey DT, Porter DL, et al. CD19-targeted chimeric antigen receptor $\mathrm{T}$-cell therapy for acute lymphoblastic leukemia. Blood. 2015; 125(26): 4017-4023, doi: 10.1182/ blood-2014-12-580068, indexed in Pubmed: 25999455.

8. Zhang L, Tang Y, Zhu X, et al. Overexpression of MiR-335-5p promotes bone formation and regeneration in mice. $\mathrm{J}$ Bone Miner Res. 2017; 32(12): 2466-2475, doi: 10.1002/jbmr.3230, indexed in Pubmed: 28846804.

9. Ji Y, Wang D, Zhang B, et al. MiR-361-3p inhibits -amyloid accumulation and attenuates cognitive deficits through targeting BACE1 in Alzheimer's disease. J Integr Neurosci. 2019; 18(3): 285-291, doi: 10.31083/j.jin.2019.03.1136, indexed in Pubmed: 31601077. 
10. Bartel DP. MicroRNAs: genomics, biogenesis, mechanism, and function. Cell. 2004; 116(2): 281-297, doi: 10.1016/s00928674(04)00045-5, indexed in Pubmed: 14744438.

11. Sandoval-Bórquez A, Polakovicova I, Carrasco-Véliz N, et al. MicroRNA-335-5p is a potential suppressor of metastasis and invasion in gastric cancer. Clin Epigenetics. 2017; 9: 114, doi: 10.1186/s13148-017-0413-8, indexed in Pubmed: 29075357.

12. Tutar Y. miRNA and cancer; computational and experimental approaches. Curr Pharm Biotechnol. 2014; 15(5): 429, doi: $10.2174 / 138920101505140828161335$, indexed in Pubmed: 25189575.

13. Nucera S, Giustacchini A, Boccalatte F, et al. miRNA-126 orchestrates an oncogenic program in b cell precursor acute lymphoblastic leukemia. Cancer Cell. 2016; 29(6): 905-921, doi: 10.1016/j.ccell.2016.05.007, indexed in Pubmed: 27300437.

14. Yang XY, Sheng Ye. miR-101 Represses T-cell acute lymphoblastic leukemia by targeting CXCR7/STAT3 axis. Oncol Res. 2019; 27(9): 997-1006, doi: 10.3727/096504018X15439 207752093, indexed in Pubmed: 30837035.

15. Huang W, Wang WT, Fang Ke, et al. MIR-708 promotes phagocytosis to eradicate T-ALL cells by targeting CD47. Mol Cancer. 2018; 17(1): 12, doi: 10.1186/s12943-018-07682, indexed in Pubmed: 29368647.

16. Iwai N, Yasui K, Tomie A, et al. Oncogenic miR-96-5p inhibits apoptosis by targeting the caspase- 9 gene in hepatocellular carcinoma. Int J Oncol. 2018; 53(1): 237-245, doi: 10.3892/ ijo.2018.4369, indexed in Pubmed: 29658604.

17. Hao H, Liu Q, Wu D, et al. Tetrahydropalmatine reduces cell death and improves functional recovery after traumatic spinal cord injury in rats. Trop J Pharm Res. 2019; 18(5): 703-711, doi: doi: http://dx.doi.org/10.4314/tjpr.v18i4.4.

18. Gao XH, Zhang YL, Zhang ZY, et al. MicroRNA-96-5p represses breast cancer proliferation and invasion through Wnt/ -catenin signaling via targeting CTNND1. Sci Rep. 2020; 10(1): 44, doi: 10.1038/s41598-019-56571-z, indexed in Pubmed: 31913290.

19. Yang CC, Chang KW. Eicosanoids and HB-EGF/EGFR in cancer. Cancer Metastasis Rev. 2018; 37(2-3): 385-395, doi: 10.1007/s10555-018-9746-9, indexed in Pubmed: 29936588.

20. Kunami N, Yotsumoto F, Ishitsuka K, et al. Antitumor effects of CRM197, a specific inhibitor of HB-EGF, in T-cell acute lymphoblastic leukemia. Anticancer Res. 2011; 31(7): 2483-2488, indexed in Pubmed: 21873163.

21. Bond J, Marchand T, Touzart A, et al. An early thymic precursor phenotype predicts outcome exclusively in HOXA-overex- pressing adult T-cell acute lymphoblastic leukemia: a Group for Research in Adult Acute Lymphoblastic Leukemia study. Haematologica. 2016; 101(6): 732-740, doi: 10.3324/haematol.2015.141218, indexed in Pubmed: 26944475.

22. Rashed WM, Hamza MM, Matboli M, et al. MicroRNA as a prognostic biomarker for survival in childhood acute lymphoblastic leukemia: a systematic review. Cancer Metastasis Rev. 2019; 38(4): 771-782, doi: 10.1007/s10555-019-09826-0, indexed in Pubmed: 31807971.

23. Zhang H, Chen R, Shao J. MicroRNA-96-5p facilitates the viability, migration, and invasion and suppresses the apoptosis of cervical cancer cells by negatively modulating SFRP4. Technol Cancer Res Treat. 2020; 19: 1533033820934132, doi: 10.1177/1533033820934132, indexed in Pubmed: 32527205.

24. Alinari L, Mahasenan KV, Yan F, et al. Selective inhibition of protein arginine methyltransferase 5 blocks initiation and maintenance of B-cell transformation. Blood. 2015; 125(16): 2530-2543, doi: 10.1182/blood-2014-12-619783, indexed in Pubmed: 25742700.

25. Mensah AA, Cascione L, Gaudio E, et al. Bromodomain and extra-terminal domain inhibition modulates the expression of pathologically relevant microRNAs in diffuse large B-cell lymphoma. Haematologica. 2018; 103(12): 2049-2058, doi: 10.3324/haematol.2018.191684, indexed in Pubmed: 30076183.

26. Wei LQ, Liang HT, Qin DC, et al. MiR-212 exerts suppressive effect on SKOV3 ovarian cancer cells through targeting HBEGF. Tumour Biol. 2014; 35(12): 12427-12434, doi: 10.1007/s13277-014-2560-2, indexed in Pubmed: 25201063.

27. Rao L, Giannico D, Leone P, et al. HB-EGF-EGFR signaling in bone marrow endothelial cells mediates angiogenesis associated with multiple myeloma. Cancers (Basel). 2020; 12(1), doi: 10.3390/cancers12010173, indexed in Pubmed: 31936715.

28. Shimura T, Yoshida M, Fukuda S, et al. Nuclear translocation of the cytoplasmic domain of HB-EGF induces gastric cancer invasion. BMC Cancer. 2012; 12: 205, doi: 10.1186/1471-240712-205, indexed in Pubmed: 22646534.

29. Tian XP, Huang WJ, Huang HQ, et al. Prognostic and predictive value of a microRNA signature in adults with T-cell lymphoblastic lymphoma. Leukemia. 2019; 33(10): 2454-2465, doi: 10.1038/s41375-019-0466-0, indexed in Pubmed: 30953029.

30. Yu S, Geng Q, Ma J, et al. Heparin-binding EGF-like growth factor and miR-1192 exert opposite effect on Runx2-induced osteogenic differentiation. Cell Death Dis. 2013; 4: e868, doi: 10.1038/cddis.2013.363, indexed in Pubmed: 24136232.

Submitted: 13 May, 2020

Accepted after reviews: 19 August, 2020 Available as AoP: 7 September, 2020 